\title{
The Process of Sentiment Analysis: A Study
}

\author{
Manasee Godsay \\ Dept. of Computer Science and Engineering, \\ Government College of Engineering Aurangabad
}

\begin{abstract}
The paper gives a detailed report of the concept-sentiment analysis and its usage. It explains the various real world applications of sentiment analysis along with the workflow that describes the execution of this analysis. The recent techniques used in the analysis have been described briefly and the appropriate performance metrics have been applied to them. The paper enlightens the need of sentiment analysis and its importance.
\end{abstract}

\section{General Terms}

Data analytics, Sentiment analysis, Opinion mining

\section{Keywords}

Sentiment analysis, polarity, lexicon, natural language processing (NLP), social media

\section{INTRODUCTION}

The term "Sentiment Analysis" itself narrates that it is analysis of the various sentiments expressed by humans over the internet, or the opinions of/feedback given by customers to various business organizations. A simple example in our day to day life where sentiment analysis comes in to picture is, when you look for the movie reviews before watching it, there are dedicated techniques available only to analyze the movie reviews [1]. On a broader note, sentimental analysis or opinion mining use data mining and natural language processing (NLP) techniques to discover, retrieve and distill information and opinions from the World Wide Web's vast textual information.[2]

Sentiment analysis allows us to track attitudes and feelings on the web. People write blog posts, comments, reviews and tweets about all sorts of different topics. We can track products, brands and people for example and determine whether they are viewed positively or negatively on the web. We can analyze: facts-"the antique was sold at a much higher price than estimated"; opinions-"the first part of the movie was better than the second one".

\section{APPLICATIONS}

\subsection{Applications in Business Intelligence}

Sentiment adds important context to social conversations. Without it, measurement of mentions alone could be misleading. If you were measuring mentions for your company's new product, you might assume a surge in mentions meant it was being well received. After all, more mentions $=$ more people talking about the product. But what if all those mentions were negative? Measuring sentiment will help you understand the overall feeling surrounding a particular subject, enabling you to create a broader and more complete picture of the social conversations that matter to you. This allows businesses to track flame detection, new product perception, brand perception, reputation management, etc. The subjective data has to be known, ex: "the design is gaudy", "customer service was prompt".

\subsection{Applications in Sociology, psychology and Political Sentiment Analysis}

It allows individuals to get an opinion on something (reviews) on a global scale like movie reviews, political views, views over a global issue, identifying child suitability of videos based on comments, bias identification in news, etc. Sentiment refers to the emotion behind a social media mention. It's a way to measure the tone of the conversationis the person happy, annoyed, angry? In politics, we can analyze trends, identify ideological bias, target advertising/messages accordingly, evaluation of public/voters' opinions. In sociology, idea propagation through groups is an important concept (cf. Rogers 1962, 'Diffusion of Innovations'), the opinions that people have and their reactions to ideas are relevant to the adoption of new ideas. In psychology, sentiment analysis provides a platform to augment psychological investigations/experiments with data extracted from NL (natural language) text. Ex: Dream sentiment analysis (Nadeau et al., 2006).

\section{THE WORKING OF SENTIMENT ANALYSIS 3.1 Challenges}

To learn the process for the execution of sentiment analysis, the challenges that accompany it should be known. The basic challenges in the process are: People express opinions in complex ways; in opinion texts, lexical content alone can be misleading; rhetorical devices/modes such as sarcasm, irony, implication, etc. are difficult to analyze.

\subsection{Content to be classified}

There are many possibilities for what we want to classifyusers of a product/service; text; sentences (paragraphs, chunks of text); predetermined descriptive phrases which are those words that can be used as a basis for classification such as groups of adjectives and nouns, adverbs and nouns, etc.; tweets, updates are other examples. Short phrases can be an important basis of classification, such as - "highest prices", "lowest quality" (both are a combination of adjective and noun, <ADJ N>).

Polarity keywords: An important factor in classification techniques is the determination of the polarity of the word, i.e. whether the word/phrase gives a positive output or a negative output. Thus, words can be grouped according to their polarity. The table below shows the results when two human subjects were asked to pick keywords that would be good indicators of sentiment polarity: 
Table 1: Comparison of accuracy for Picking Keywords for Indicating Polarity

\begin{tabular}{|l|l|l|l|}
\hline & PROPOSED WORD LIST & ACCURACY & TIES \\
\hline HUMAN 1 & $\begin{array}{l}\text { Pos: dazzling, brilliant, phenomenal, excellent, fantastic. } \\
\text { Neg: suck, terrible, awful, unwatchable, hideous }\end{array}$ & $58 \%$ & $75 \%$ \\
\hline HUMAN 2 & $\begin{array}{l}\text { Pos: gripping, mesmerizing, riveting, spectacular, cool, } \\
\text { awesome, thrilling, badass, excellent, moving, exciting } \\
\text { Neg: bad, clichéd, sucks, boring, stupid, slow }\end{array}$ & $64 \%$ & $39 \%$ \\
\hline STATISTICS-BASED & $\begin{array}{l}\text { Pos: love, wonderful, best, great, superb, still, beautiful } \\
\text { Neg: bad, worst, stupid, waste, boring, ?, }\end{array}$ & $69 \%$ & $16 \%$ \\
\hline
\end{tabular}

[Values taken from Pang et al. (2002)]

$\mathrm{Hu}$ and Liu, KDD-2004 have published an "opinion lexicon" which categorizes approximately 6800 words as positive or negative and which can be downloaded from the link: http://www.cs.uic.edu/ liub/fbs/sentiment-analysis.html

The steps involved in sentiment analysis can be explained with the help of a flowchart, as shown below.

- Firstly, the goal is to be set, which includes determining the sentiment analysis goal and the scope for the text content.

- Secondly, text processing has to be done which involves determining the source i.e. whether you are taking the data from web, micro-blogging site, etc. The text then has to be loaded to the processing system (the system, technique to be used for the analysis), unwanted words from the text are deleted and organizing the emotional symbols that people use in texts into words. Also, it observed that to express strong sentiments, uppercase alphabets are used (such as OUTRAGEOUS!)

- Then, comes parsing the content which involves segmenting the words based on their polarity; tagging the parts of speech used (adjective, noun, etc.); identifying the terms.

- To ensure the correct analysis text refinement should be, that is finding the stop words and synonyms, etc.

- The last step is analysis and scoring: It involves determining the sentiments bearing phrases from the data and scoring them. Scoring is the process in which the intensity of the sentiment is analyzed. An example for scoring is shown in the table below:

Table 2: Sample scores for sentiments:

\begin{tabular}{|l|l|}
\hline SCORE & TEXT \\
\hline 2 & You're awesome and I love you \\
\hline-5 & I hate and hate and hate. So angry. Die! \\
\hline 4 & $\begin{array}{l}\text { Impressed and amazed: you are peerless } \\
\text { in your achievement of unparalleled } \\
\text { mediocrity. }\end{array}$ \\
\hline
\end{tabular}

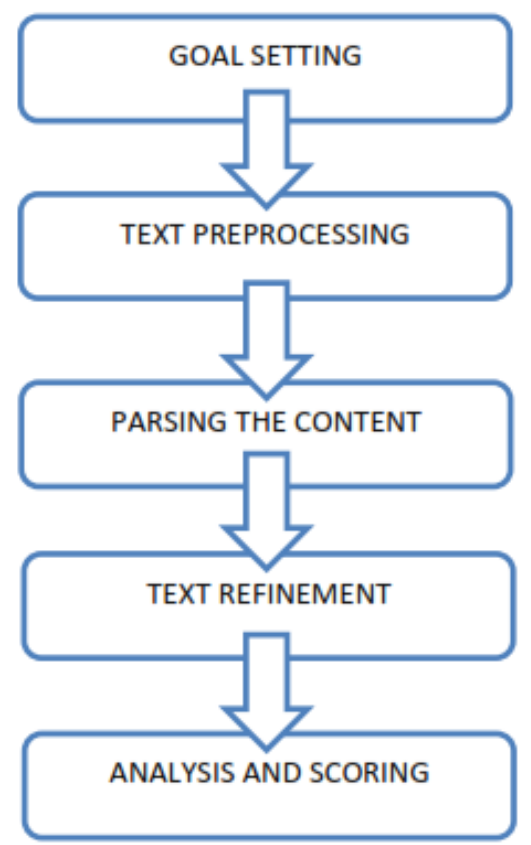

Fig. 2.1: General Workflow of the Sentiment Analysis Process

\subsection{TECHNIQUES USED FOR SENTIMENT ANALYSIS}

Broadly, there exist two types of methods for sentiment analysis: 1. machine-learning-based and 2. lexical-based methods.

Machine learning methods often rely on supervised classification approaches, where sentiment detection is framed as a binary (i.e., positive or negative). This approach requires labeled data to train classifiers [3]. While one advantage of learning-based methods is their ability to adapt and create trained models for specific purposes and contexts, their drawback is the availability of labeled data and hence the low applicability of the method on new data. This is because labeling data might be costly or even prohibitive for some tasks. On the other hand, lexical-based methods make use of a predefined list of words, where each word is associated with a specific sentiment. The lexical methods vary according to the context in which they were created. The available research shows machine learning approaches (Naive Bayes, Maximum Entropy, and SVM) to be more suitable for Twitter than the lexical-based LIWC method [4]. Similarly, classification 
methods (SVM, and Multinomial Naive Bayes) are more suitable than SentiWordNet for Twitter [5].

A brief description of certain methods that are widely used for different contexts in sentiment analysis which cover diverse techniques such as the use of Natural Language Processing (NLP) in assigning polarity, the use of Amazon's Mechanical Turk (AMT) to create labeled datasets, the use of psychometric scales to identify mood-based sentiments, the use of supervised and unsupervised machine learning techniques, etc. is explained as follows:

\subsubsection{Emoticons}

The simplest to detect the way polarity (i.e., positive and negative affect) of a message is based on the emoticons it contains. Emoticons have become popular in recent years, to the extent that some (e.g. <3) are now included in English Oxford Dictionary [6]. Emoticons are primarily face-based and represent happy or sad feelings, although a wide range of non-facial variations exist: for instance, $<3$ represents a heart and expresses love or affection. As one may expect, the rate of messages containing at least one emoticon is very low compared to the total number of messages that could express emotion. A recent work has identified that this rate is less than $10 \%$ [7]. Therefore, emoticons have been often used in combination with other techniques for building a training dataset in supervised machine learning techniques [8].

\subsubsection{LIWC}

Linguistic Inquiry and Word Count [4] is a text analysis tool that evaluates emotional, cognitive, and structural components of a given text based on the use of a dictionary containing words and their classified categories. In addition to detecting positive and negative affects in a given text, LIWC provides other sets of sentiment categories. For example, the word "agree" belongs to the following word categories: assent, affective, positive emotion, positive feeling, and cognitive process.

\subsubsection{SentiStrength}

Machine-learning-based methods are suitable for applications that need content-driven or adaptive polarity identification models. One of the effective works by M. Thelwall, "Heart and soul: Sentiment strength detection in the social web with sentistrength", compared a wide range of supervised and unsupervised classification methods, including simple logistic regression, SVM, J48 classification tree, JRip rule-based Happiness Index [16] is a sentiment scale that uses the popular Affective Norms for English Words (ANEW) [17]. ANEW is a collection of 1,034 words commonly used associated with their affective dimensions of valence, arousal, and dominance. Happiness Index was constructed based on the ANEW terms and has scores for a given text between 1 and 9 , indicating the amount of happiness existing in the text. The authors calculated the frequency that each word from the ANEW appears in the text and then computed a weighted average of the valence of the ANEW study words. The validation of the Happiness Index score is based on examples. In particular, the authors applied it to a dataset of song lyrics, song titles, and blog sentences. They found that the happiness score for song lyrics had declined from 1961 to 2007, while the score for blog posts in the same period had increased.

\subsubsection{PANAS- $t$ :}

The PANAS-t [18] is a psychometric scale proposed by a comprehensive work "Comparing and Combining Sentiment Analysis methods" [19]. The method consists of an adapted version of the Positive Affect Negative Affect Scale (PANAS) classifier, SVM regression, AdaBoost, Decision Table, Multilayer Perception, and Na"ive Bayes. The core classification of this work relies on the set of words in the LIWC dictionary [4], and the authors expanded this baseline by adding new features such as, adding list of booster words (such as "very"), a list of emoticons, repeated punctuation ("Cool!!!"). The authors have released a tool SentiStrength, which uses a combination of learning techniques that produce comparatively effective and accurate results.[9]

\subsubsection{SentiWordNet}

SentiWordNet[10] is a tool used in opinion mining and is based on English lexical dictionary WordNet[11]. The dictionary groups adjectives and nouns and other parts of speech in sets with similar meaning words called 'synsets'(Synonym sets). SentiWordNet does the scoring of the words (as we saw in the process of Sentiment Analysis) from the sets to indicate the sentiment of the test as : positive, negative, neutral.

\subsubsection{SenticNet}

SenticNet[12] is a method that involves artificial intelligence and semantic Web techniques. The goal of SenticNet is to provide the polarity of common sense concepts from natural language text at a semantic level, instead of the syntactic level. The method uses Natural Language Processing (NLP) techniques to create a polarity for nearly 14,000 concepts. For instance, to interpret a message "Excellent, camera lens!", SenticNet first tries to identify concepts, which are "excellent" and "camera lens" in this case.

Then it assigns values i.e. the polarity scores $\mathrm{x}$ and $\mathrm{y}$ to "excellent" and "camera lens" respectively, and the result is $\mathrm{x}+\mathrm{y}$. SenticNet was tested and evaluated as a tool to measure the level of polarity in opinions of patients about the National Health Service in England [13].

\subsubsection{SASA}

SailAil Sentiment Analyzer (SASA) [14]. SASA is a method based on machine learning techniques such as SentiStrengh and was evaluated with 17,000 labeled tweets on the 2012 U.S. Elections. The open source tool was evaluated by the Amazon Mechanical Turk (AMT) [15], where "turkers" were invited to label tweets as positive, negative, neutral, or undefined.

\subsubsection{Happiness Index:}

[20], which is a well-known method in psychology. The PANAS-t is based on a large set of words associated with eleven moods: joviality, assurance, serenity, surprise, fear, sadness, guilt, hostility, shyness, fatigue, and attentiveness. The method is designed to track any increase or decrease in sentiments over time. To associate text to a specific sentiment, PANAS-t first utilizes a baseline or the normative values of each sentiment based on the entire data. Then the method computes the $\mathrm{P}(\mathrm{s})$ score for each sentiment $\mathrm{s}$ for a given time period as values between $[-1.0,1.0]$ to indicate the change.[19]

\subsection{METRICS FOR SENTIMENT ANALYSIS}

The authors [19] have done a comparison of the methods to test the performance of the methods using the following metrics:

In order to define the metrics used to evaluate the methods, the work considered the following metrics (table 3 ): 
Using the above metrics a sample analysis on H1N1 data was done [3]. It shows the result for the outbreak of the H1N influenza, a worldwide epidemic declared by the World Health Organization in 2009. In this case, SentiWordNet and SenticNet have the highest coverage with $95 \%$ and $93 \%$, respectively, followed by SentiStrength with $61 \%$. [19]:

\begin{tabular}{|l|l|ll|}
\hline \multicolumn{2}{|l|}{} & \multicolumn{2}{|l|}{$\begin{array}{l}\text { Actual observation } \\
\text { Positive: Negative: }\end{array}$} \\
\hline $\begin{array}{l}\text { Predicted } \\
\text { Expectation: }\end{array}$ & Positive: & a & b \\
& Negative: & c & d \\
\hline
\end{tabular}

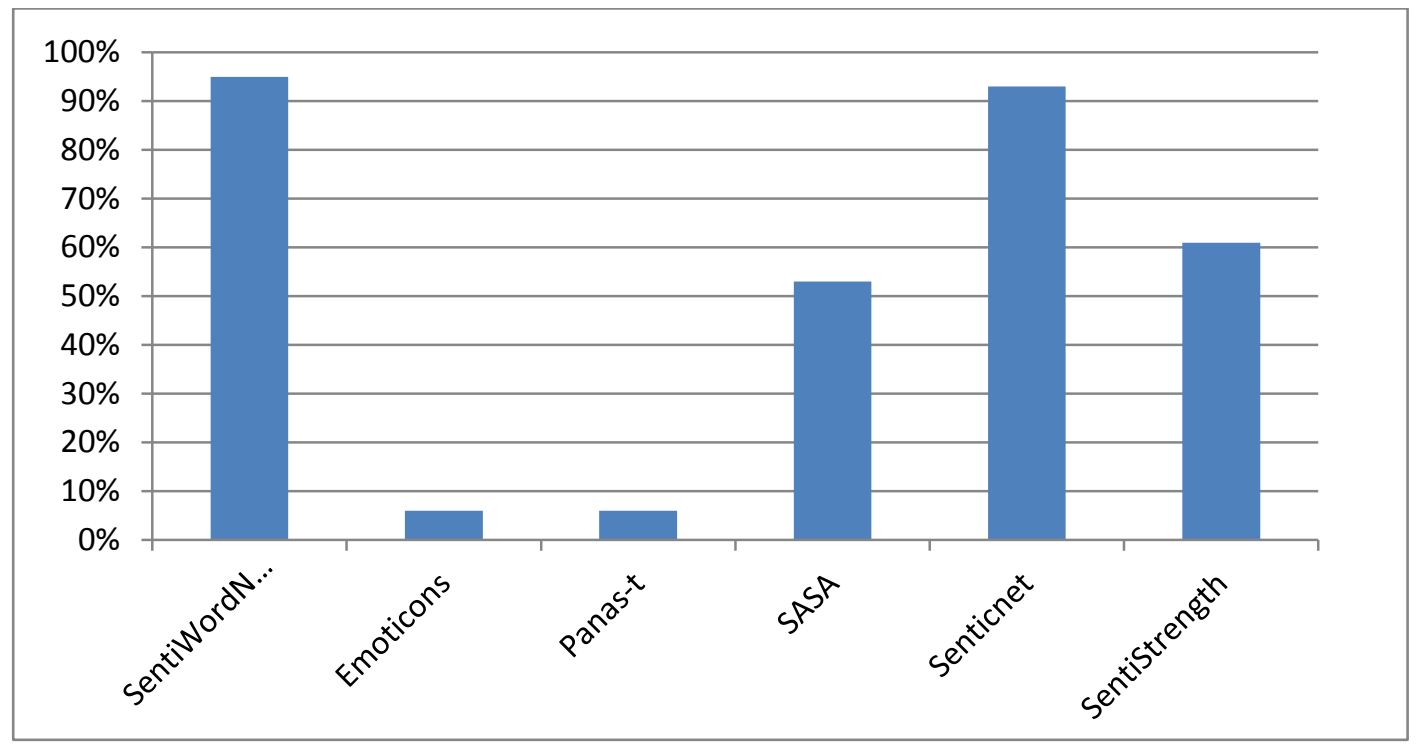

Fig. 2: Graph for Performance of the Eight Methods Mentioned above on H1N1 Data [3]

\section{CONCLUSION}

The Web has changed from "read-only" to "read-write"[2], thus, opening doors to the collection of enormous data that portrays human opinions and sentiments. The need of sentiment analysis arises here to gain collective results on what the masses express. The classification of methods in sentiment analysis show that the technique majorly depends on what kind of data is being analyzed and thus every type has its own suitable technique. The future sentiment analysis and opinion mining techniques need wider common-sense approaches and are to be inspired by human thought process so that the results are more honest, accurate and parallel to the human psychology.

\section{ACKNOWLEDGEMENTS}

I would like thank Mrs. M.B. Nagori(asst. prof, Dept. of computer science, Government College of Engineering, Aurangabad) for her guidance and for providing a deep insight into the topic, such that the study could be carried out.

\section{REFERENCES}

[1] A Comparison of Sentiment Analysis Techniques: Polarizing Movie Blogs, Michelle Annett and Grzegorz Kondrak

[2] Erik Cambria , National University of Singapore, Bjorn Schuller, Technical University of Munich, Yunqing Xia , Tsinghua University, Catherine Havasi, Massachusetts Institute of Technology, New Avenues in Opinion Mining and Sentiment Analysis, Issue No.02 - MarchApril (2013 vol.28) pp: 15-21; Published by the IEEE Computer Society.

[3] B. Pang, L. Lee, and S. Vaithyanathan. Thumbs up?: sentiment classification using machine learning techniques. In ACL Conference on Empirical Methods in Natural Language Processing, pages 79-86, 2002.

[4] Y. R. Tausczik and J. W. Pennebaker. The psychological meaning of words: Liwc and computerized text analysis methods. Journal of Language and Social Psychology, 29(1):24-54, 2010

[5] A. Bermingham and A. F. Smeaton. Classifying Sentiment in Microblogs: Is Brevity an Advantage? In ACM International Conference on Information and Knowledge Management (CIKM), pages 1833-1836, 2010.

[6] OMG! Oxford English Dictionary grows a heart: Graphic symbol for love (and that exclamation) are added as words. tinyurl.com/klv36p.

[7] J. Park, V. Barash, C. Fink, and M. Cha. Emoticon style: Interpreting differences in emoticons across cultures. In International AAAI Conference on Weblogs and Social Media (ICWSM), 2013.

[8] J. Read. Using emoticons to reduce dependency in machine learning techniques for sentiment classification. In ACL Student Research Workshop, pages 43-48, 2005.

[9] M. Thelwall. Heart and soul: Sentiment strength detection in the social web with sentistrength. http://sentistrength.wlv.ac.uk/documentation/ SentiStrengthChapter.pdf.

[10] Esuli and Sebastiani. Sentwordnet: A publicly available lexical resource for opinion mining. In International Conference on Language Resources and Evaluation (LREC), pages 417-422, 2006. 
[11] G. A. Miller. Wordnet: a lexical database for english. Communications of the ACM, 38(11):39-41, 1995.

[12] E. Cambria, R. Speer, C. Havasi, and A. Hussain. Senticnet: A publicly available semantic resource for opinion mining. In AAAI Fall Symposium Series, 2010.

[13] E. Cambria, A. Hussain, C. Havasi, C. Eckl, and J. Munro. Towards crowd validation of the uk national health service. In ACM Web Science Conference (WebSci), 2010.

[14] H. Wang, D. Can, A. Kazemzadeh, F. Bar, and S. Narayanan. A system for real-time twitter sentiment analysis of 2012 u.s. presidential election cycle. In ACL System Demonstrations, pages 115-120, 2012.

[15] Amazon. Amazon mechanical turk. https://www.mturk.com/. Accessed June 17, 2013.

[16] P. S. Dodds and C. M. Danforth. Measuring the happiness of large-scale written expression: songs, blogs, and presidents. Journal of Happiness Studies, 11(4):441456, 2009.

[17] M. M. Bradley and P. J. Lang. Affective norms for English words (ANEW): Stimuli, instruction manual, and affective ratings. Technical report, Center for Research in
Psychophysiology, University of Florida, Gainesville, Florida, 1999.

[18] P. Gonc salves, F. Benevenuto, and M. Cha. PANAS-t: A Pychometric Scale for Measuring Sentiments on Twitter. abs/1308.1857v1, 2013.

[19] Pollyanna Gonçalves, Matheus Araújo, Fabrício Benevenuto, Meeyoung Cha, Comparing and Combining Sentiment Analysis Methods, arXiv:1406.0032v1 [cs.CL] 30 May 2014

[20] D. Watson and L. Clark. Development and validation of brief measures of positive and negative affect: the panas scales. Journal of Personality and Social Psychology, 54(1):1063-1070, 1985.

[21] https://sivaanalytics.wordpress.com/2013/10/10/sentimen t-analysis-on-twitter-data-using-r-part-i/

[22] www.lctmaster.org/files/MullenSentimentCourseSlides.pdf

[23] Walaa Medhata, Ahmed Hassanb, Hoda Korashyb, Ain Shams, Sentiment analysis algorithms and applications: A survey. Engineering journal, volume 5, issue 4, December 2014, Pages 1093-1113. 